\title{
Light-induced transmission nonlinearities in gallium selenide
}

\author{
Ch. Ferrer-Roca, ${ }^{\text {a) }}$ J. Bouvier, ${ }^{\text {b) }}$ A. Segura, M. V. Andrés, and V. Muñoz \\ Institut de Ciéncia dels Materials, Departament de Física Aplicada, Universitat de València, \\ Dr. Moliner 50, 46100 Burjassot (Valencia), Spain
}

(Received 1 June 1998; accepted for publication 5 January 1999)

\begin{abstract}
The intensity of a He-Ne laser $(633 \mathrm{~nm}, 5 \mathrm{~mW})$ transmitted by different GaSe samples is observed to change in correlation with a Nd-yttrium-aluminum-garnet laser pulse $(532 \mathrm{~nm}, 7.8 \mathrm{~ns}, 3 \mathrm{~mJ})$ which excites them. Such time response has been attributed to a nonlinear optical effect, i.e., a decrease in the refractive index due to the exciton screening by the photogenerated carriers. A calculation of the absorption coefficient and refractive index at different carrier concentrations has led to a reconstruction of transmittance transients which fully agree with the experimental data at different incident intensities and temperatures. (C) 1999 American Institute of Physics.

[S0021-8979(99)05707-2]
\end{abstract}

\section{INTRODUCTION}

Layered III-VI semiconductors and among them gallium selenide $(\mathrm{GaSe})$ are known to present outstanding nonlinear optical properties which have been widely investigated during the last few years. Their refractive index anisotropy makes them suitable for second harmonic generation, ${ }^{1-6}$ parametric oscillation, ${ }^{7}$ and frequency mixing ${ }^{8-10}$ in the middle infrared spectral zone where they are transparent.

On the other hand, the optical properties can be varied significantly by exciting the samples with a pump laser beam. This fact, combined with feedback, lead to optical bistability and hence to the possibility of reproducing the operation modes of electronic devices by purely optical means. Such variations of the absorption coefficient and refractive index can be very large in the vicinity of the absorption edge and they are nonlinear because they do not depend directly on the incident beam intensity, but on some other variable which depends on it. These optical effects have been reported in cadmium sulfide $(\mathrm{CdS})^{11}$ and $\mathrm{GaSe}^{12-14}$ and they are explained in terms of the screening of excitons by photogenerated carriers. The collision broadening of the free exciton resonance has also been claimed in order to explain changes in the absorption and refractive index in $\mathrm{CdS}$ and GaSe. ${ }^{15}$

Recently, a new nonlinear optical effect has been shown to occur in gallium and indium selenides, corresponding to transitions from a lower-energy valence band to the uppermost one when a nonequilibrium degenerate hole gas is created in the latter by a laser pulse. ${ }^{16}$

The aim of this article is to evidence, in a quantitative way, the nonlinear nature of variations in the measured transmittance in GaSe when samples are optically excited. After a description of the experiment we present the transmission pulses obtained at different temperatures and incident intensities, together with some qualitative considerations on their characteristics. Then we use a semiphenomenological model

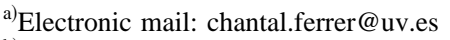

${ }^{b}$ ESPCI, Laboratoire de Physique du Solide, 10 Rue Vauquelin, 75231 Paris, Cedex 05, France.
}

to calculate the absorption coefficient which allows to reproduce the transmission time response.

\section{EXPERIMENT}

The GaSe crystals $\left(E_{g}=2.02 \mathrm{eV}\right)$ used in this study have been grown by the Bridgman-Stockbarger method from a stoichiometric polycrystalline melt. The quality of the crystals was tested through the temperature dependence of the exciton absorption peak width. The full width at half maximum (FWHM) of the exciton exhibits a lattice-controlled dependence in the range from 300 to $77 \mathrm{~K}$ and attains at 77 $\mathrm{K}$ a value of the order of $5-6 \mathrm{meV}$, as reported in the literature for high-purity samples. ${ }^{17,18}$

Samples with mirrorlike surfaces parallel to the layers were cleaved from the ingots with a razor blade and cut into slabs of $4 \times 4 \mathrm{~mm}^{2}$ in size and $2-30 \mu \mathrm{m}$ thick, being the crystal anisotropy axis ( $c$ axis) perpendicular to the layers. Samples were mounted in an aluminum holder with several holes and stuck to it with silver paste in order to improve the thermal contact. The sample holder could be heated up to $100^{\circ} \mathrm{C}$. The optical setup was similar to that used in a previous work. ${ }^{16} \mathrm{~A} \mathrm{He}-\mathrm{Ne}$ laser $(633 \mathrm{~nm}, 5 \mathrm{~mW})$ generates the probe beam which crosses the sample parallel to the $c$ axis and reaches a Si photodiode of about $10^{4} \mathrm{~V} / \mathrm{W}$ gain and a dc $125 \mathrm{MHz}$ nominal frequency band. The photoreceiver output is monitored by a computer-controlled $400 \mathrm{MHz}$ bandwidth oscilloscope. Signal to noise ratio was improved by using the averaging capabilities of the oscilloscope.

The second harmonic of Nd-yttrium-aluminum-garnet (YAG) laser $(532 \mathrm{~nm})$ worked as a pump laser, providing optical pulses of $7.8 \mathrm{~ns}$ FWHM and a $10 \mathrm{~Hz}$ repetition rate. The beam diameter was $3 \mathrm{~mm}$ and the energy impinging on the sample was limited to $3 \mathrm{~mJ}$ per pulse in most experiments, corresponding to a photon flux on the order of 1.7 $\times 10^{25} \mathrm{ph} \mathrm{cm}^{-2} \mathrm{~s}^{-1}$ at the pump pulse maximum. The residual pump signal was suppressed by an optical filter.

\section{EXPERIMENTAL RESULTS AND DISCUSSION}

The $\mathrm{He}-\mathrm{Ne}$ probe beam intensity transmitted by GaSe samples was observed to change in coincidence with the $\mathrm{Nd}$ - 

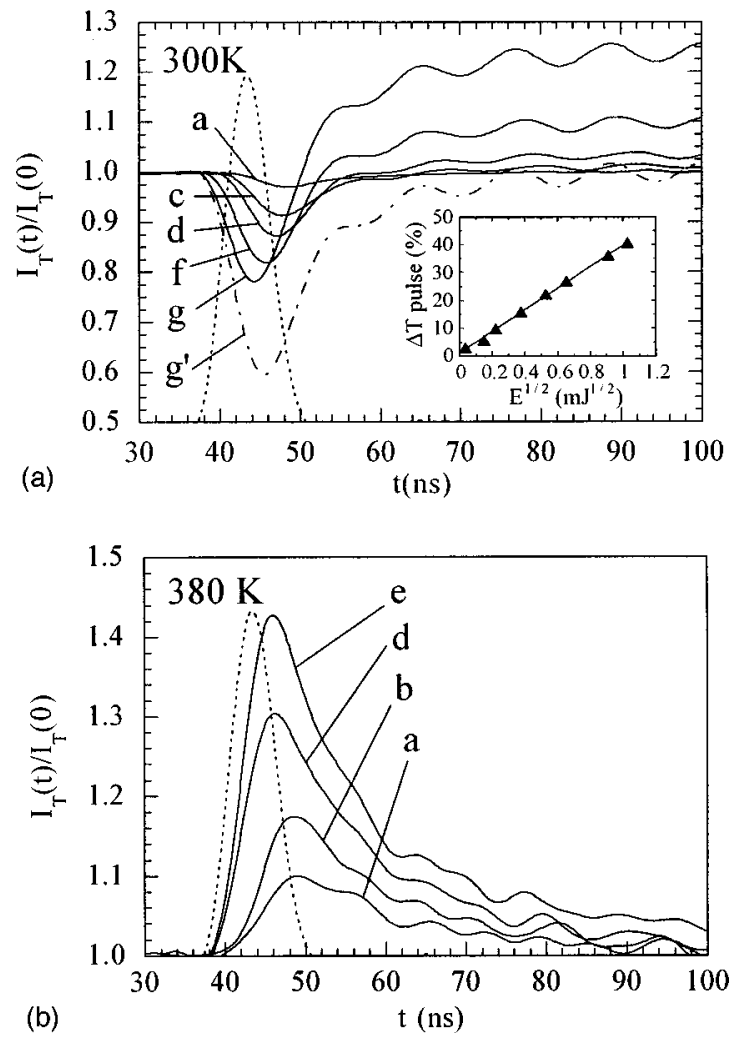

FIG. 1. (a) Time response of a $15 \mu \mathrm{m}$ sample transmitted intensity, at 300 $\mathrm{K}$, relative to the value in the absence of excitation at different incident pump photon fluxes (solid line curves). The dash-and-dot curve $\mathrm{g}^{\prime}$ is the transmission signal $g$ after the subtraction of the thermal contribution. The inset shows the transmittance corrected for thermal effects vs the square root of the absorbed incident energy. The dashed line curve (---) is the pump incident laser pulse. (b) The same at $380 \mathrm{~K}$.

YAG (pump beam) incident pulses. The pump energies ranged from 0.15 to $3 \mathrm{~mJ}$ and, specifically, the following photon fluxes were considered $\left(\mathrm{cm}^{-2} \mathrm{~s}^{-1}\right)$ : (a) $0.85 \times 10^{24}$, (b) $1.4 \times 10^{24}$, (c) $2.3 \times 10^{24}$, (d) $3.6 \times 10^{24}$, (e) $5.4 \times 10^{24}$, (f) $8.5 \times 10^{24}$, and $(\mathrm{g}) 17 \times 10^{24}$. Throughout this article, the experimental curves will be labeled with these lower-case letters in order to indicate the pump photon flux at which they have been obtained.

The solid lines in Fig. 1(a) show a typical set of intensity versus time curves for the probe beam transmitted by a 15$\mu \mathrm{m}$-thick GaSe sample when excited by pump pulses at different energies, at $300 \mathrm{~K}$. The transient response has been normalized to the transmission value in the absence of excitation. The negative 10-ns-wide pulse which appears practically in coincidence with the pump pulse implies a decrease of the transmitted intensity which can be as high as $78 \%$ of the initial value for the maximum pump energy. After this pulse, in a time interval of about $20 \mathrm{~ns}$, the transmitted signal increases and reaches a quasisteady state which lasts several tens of microseconds. This relatively slow transient exhibits a superimposed oscillation at a definite frequency. The same characteristics can be observed in Fig. 1(b), which shows the transmission time response at $380 \mathrm{~K}$ and at different pump laser energies. In this case the transient is positive and represents an increment up to $40 \%$ over the initial value, whereas both the steady state level and the amplitude of the oscillations are lower.

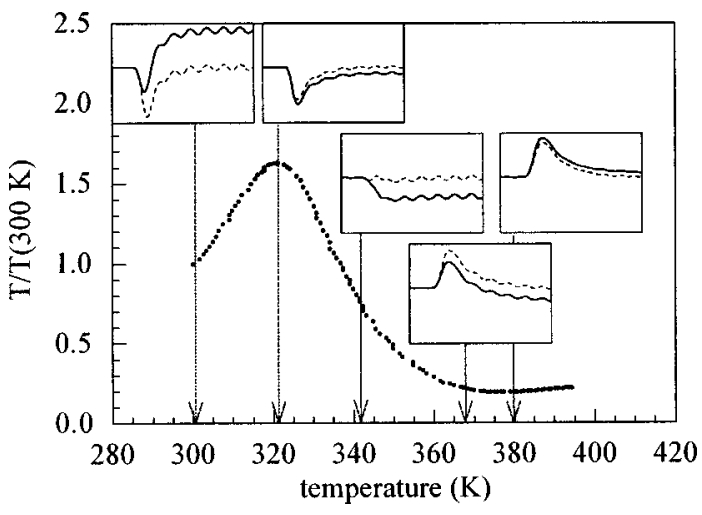

FIG. 2. Steady state transmission $(T)$ vs the sample temperature relative to its value at $300 \mathrm{~K}$. The inset graphs show the transmission pulses under excitation at a constant photon flux (nominal $17 \times 10^{24} \mathrm{~cm}^{-2} \mathrm{~s}^{-1}$ ) and at the temperatures indicated by the arrows, before (solid lines) and after (dashed lines) the correction for thermal effects.

The sinusoidal modulation has been studied elsewhere ${ }^{19}$ and can be explained in terms of photoacoustic generation of dilatational waves which become resonant within the sample leading to a variation of the optical path and hence of the sample transmittance.

The change in the quasisteady state of the transmittance after a pump pulse has a thermal origin and has been also reported. ${ }^{18,19}$ The pump energy is deposited in the sample and its temperature is raised, leading to an increase in the refractive index. On the assumption that thermal changes are proportional to the pump pulse total energy absorbed by the sample until a given instant, they can be straightforwardly calculated and subtracted from the experimental transient in order to isolate the fast contribution. In our example at $300 \mathrm{~K}$ such subtraction implies a corrected transmission transient which represents $60 \%$ of the initial value for the maximum pump energy [curve $g^{\prime}$ in Fig. 1(a)], in which the thermal part of the transmittance has already been subtracted.

The discrete points in Fig. 2 show the experimental transmittance in the absence of excitation versus temperature. As explained in more detail in a previous work, ${ }^{19}$ the samples exhibit a Fabry-Pérot transmittance [Eq. (1)], in which the refractive index and the absorption coefficient increase with temperature. The inset figures are transmittance pulses under excitation $(3 \mathrm{~mJ})$ at different temperatures before (solid lines) and after (dashed lines) the subtraction of the thermal contribution. The thermal steady state transmittance increment or decrement, which can be observed in the different inset figures, is consistent with the slope of the transmittance-versus-temperature dotted curve.

The thermal energy actually absorbed by the sample can be easily obtained from the temperature increment (for $\mathrm{GaSe}$ $C=48.5 \mathrm{~J} / \mathrm{mol} / \mathrm{K}$ and $\left.\rho=5.03 \mathrm{~g} / \mathrm{cm}^{3}\right),{ }^{20}$ and in the case of a nominal pump energy of $3 \mathrm{~mJ}$, the quantity absorbed by the sample is $1.06 \mathrm{~mJ}$, equivalent to a photon flux of 6 $\times 10^{24} \mathrm{~cm}^{-2} \mathrm{~s}^{-1}$. Once the transients have been corrected by removing the thermal contribution and the impinging energy has been recalculated in order to obtain the absorbed part, the maximum transmittance decrement is found to keep a linear dependence on the square root of the absorbed energy, as shown in the inset in Fig. 1(a). 
Such "electronic contribution"- as we will call the corrected transmission from now on-can be accounted for by nonlinear changes in the absorption coefficient $(\alpha)$ and refractive index $(n)$ caused by the photogeneration of carriers. Let us discuss why, as is shown in Fig. 2, the electronic contribution is negative at low temperatures, disappears at $341 \mathrm{~K}$, and then reappears as a positive pulse at higher temperatures. As we previously pointed out, the transmittance is that of a Fabry-Pérot

$$
T=\frac{(1-R)^{2} e^{-\alpha d}}{\left(1-\mathrm{Re}^{-\alpha d}\right)^{2}} \frac{1}{1+F \sin ^{2} \delta} ;
$$

with

$$
F=\frac{4 \mathrm{Re}^{-\alpha d}}{\left(1-\mathrm{Re}^{-\alpha d}\right)^{2}} ; \quad \delta=\frac{4 \pi n d}{\lambda} .
$$

The transmission relative variation with both the refractive and absorptive contributions is therefore

$$
\begin{aligned}
\frac{\Delta T}{T}= & \left(-\frac{F \delta \sin 2 \delta}{\left(1+F \sin ^{2} \delta\right) n}\right) \Delta n \\
& +\left(-\frac{1+\mathrm{Re}^{-\alpha d}}{1-\mathrm{Re}^{-\alpha d}} \frac{d}{1+F \sin ^{2} \delta}\right) \Delta \alpha .
\end{aligned}
$$

The term in parentheses in the absorptive contribution is always negative whereas the refractive one depends on the $\operatorname{sign}$ of $\sin 2 \delta$. At $300 \mathrm{~K}$ the absorption coefficient at the probe wavelength is very low $\left(\approx 36 \mathrm{~cm}^{-1}\right)$ and the transmittance variation is dominated by the refractive index changes, so we can just concentrate on the first term in Eq. (2). At 300 $\mathrm{K}$ and for a $d=15.047 \mu \mathrm{m}$ the term $\sin 2 \delta$ is negative, hence a decrement in the refractive index also leads to a decrement in the transmission. In our example the observed maximum transmission decrement is consistent with a change in the refractive index $\Delta n=-0.001$.

At $380 \mathrm{~K}$ the absorption coefficient increases due to the shift of the absorption edge and the excitonic peak towards the probe wavelength. The changes in the absorption coefficient become thus more relevant, leading to a predominance of the second term in Eq. (2) and hence to a positive transmission pulse. At this temperature the transmission increment is consistent with a change in the absorption coefficient $\Delta \alpha \approx-300 \mathrm{~cm}^{-1}$. At any intermediate temperature the opposite effects of the absorptive and refractive contributions may lead to a total suppression of the electronic peak as observed at $341 \mathrm{~K}$.

In the next section we will confirm the nonlinear origin of the observed transmission peaks. The absorption coefficient will be calculated with the aid of the model developed by Bányai and $\mathrm{Koch}^{21}$ and, together with the refractive index calculus via the Kramers-Krönig relations, we will reproduce the transmission time response at different pump intensities and temperatures.

\section{CALCULATION OF THE TRANSMISSION ELECTRONIC CONTRIBUTION}

As pointed out in the preceding section, the semiconductor nonlinearities are mainly caused by the elevated concen- tration of electrons and holes which are created by the absorption of the exciting laser light. Such carriers, which are assumed to be in quasiequilibrium in their respective bands, are responsible for the plasma screening of the Coulomb interaction between electrons and holes (which reduces the exciton binding energy and shifts the semiconductor gap) and for the modification of the band filling factor. Bányai and Koch $^{21}$ developed a partly phenomenological theory in which such effects are incorporated into a generalized Elliott formula for the absorption through a density-dependent parameter $g=12 / \pi^{2} a_{0} \kappa, a_{0}$ being the Bohr radius and $\kappa$ the screening wave number given by

$$
\begin{aligned}
\kappa^{2}= & \frac{4}{\pi a_{0}^{2}}\left(\frac{k_{B} \theta}{E_{R}}\right)^{1 / 2} \int_{0}^{\infty} d x x^{1 / 2} \sum_{\alpha=e, h}\left(\frac{m_{\alpha}}{m_{\mathrm{ex}}}\right)^{3 / 2} \\
& \times f\left(x-\mu_{\alpha}\right)\left[1-f\left(x-\mu_{\alpha}\right)\right],
\end{aligned}
$$

where $\theta$ is the temperature, $E_{R}$ is the exciton Rydberg energy, $m_{\mathrm{ex}}$ is the exciton reduced mass, $m_{\alpha}$ and $\mu_{\alpha}$ the carrier mass and quasi-Fermi level, respectively ( $e$ : electrons, $h$ : holes), and $f$ is the Fermi-Dirac distribution function.

The exciton wave functions obey a modified Wannier equation with a screened Coulomb potential and a band gap shift. The screened Coulomb potential, approximated by the Hulthén potential, is

$$
V_{H}=-\frac{2 e}{\epsilon_{0} a_{0} g}\left[e^{2 r / a_{0} g}-1\right]^{-1}
$$

and the band gap shift

$$
\delta E_{g}=\left\{\begin{array}{l}
-1+\left(1-\frac{1}{g}\right)^{2} E_{R} \quad g \geqslant 1 \\
-\frac{1}{g} E_{R} \quad g<1
\end{array},\right.
$$

where an excitation-independent exciton energy has been assumed.

Bányai and Koch solved the Wannier equation and arrived at the following expression for the absorption coefficient:

$$
\begin{aligned}
\alpha(\omega)= & \alpha_{0} A(\omega)\left\{\sum_{l=1}^{\left[g^{1 / 2}\right]} \pi \delta_{\Gamma}\left(\widetilde{\omega}+(1 / l-l / g)^{2}\right)\right. \\
& \times \frac{2\left(g-l^{2}\right)\left(2 l^{2}-g\right)}{l^{3} g^{2}} \prod_{\substack{n=1 \\
(n \neq l)}}^{\infty} \frac{n^{2}\left[n^{2} l^{2}-\left(g-l^{2}\right)^{2}\right]}{\left(n^{2}-l^{2}\right)\left(n^{2} l^{2}-g^{2}\right)} \\
& +\int_{0}^{\infty} d x x^{1 / 2} \prod_{n=1}^{\infty}\left(1+\frac{2 g n^{2}-g^{2}}{\left(n^{2}-g\right)^{2}+n^{2} g^{2} x}\right) \\
& \left.\times \delta_{\Gamma}(x-\widetilde{\omega})\right\},
\end{aligned}
$$

where $\alpha_{0}$ is proportional to the absolute square of the interband transition matrix, and $A(\omega)=\tanh \left(\left(\hbar \omega-\mu_{e}\right.\right.$ $\left.\left.-\mu_{h}\right) / 2 k_{B} T\right)$ is the band filling factor, $\widetilde{\omega}=\left(\hbar \omega-E_{g}\right) / E_{R}$, $\delta_{\Gamma}(x)=E_{R} / \pi \Gamma \cosh \left(x E_{R} / \Gamma\right)$ is the exciton line shape function of width $\Gamma$, and $E_{g}=E_{g}^{0}+\delta E_{g}$ is the renormalized gap 
energy which depends on the carrier density. The sum runs over the existing bound states up to the largest integer number. If the carrier density is greater than the Mott density, $g<1$ and the sum vanishes, so that only the contribution of the continuum, represented by the integral, is significant.

We have used expression (6) in a code which first calculates the quasi-Fermi levels by taking the carrier density as an input, then calculates the screening wave number $\kappa$ (or, equivalently, the parameter $g$ ) and the gap renormalization and finally the absorption coefficient as a function of frequency. In GaSe the perpendicular and parallel effective masses of electrons and holes $\operatorname{are}^{22} m_{e \perp}=0.15 m_{0}, m_{e \|}$ $=0.26 m_{0}, m_{h \perp}=0.71 m_{0}$, and $m_{h \|}=0.17 m_{0}$, the perpendicular and parallel permittivities ${ }^{20} \epsilon_{0 \perp}=10.6, \epsilon_{0 \|}=6.18$, $E_{R}=19.2 \mathrm{meV}$ and $^{20} E_{g}^{0}=2.02-4.5 \times 10^{-4}(T-300)$. The $\delta$ function width is, for $\mathrm{GaSe}^{22} \quad \Gamma=10.5-0.055(300$ $-\theta) \mathrm{meV}$. $\Gamma$ has been considered excitation independent.

Figures 3(a) and 4(a) show the calculated absorption coefficient at 300 and $380 \mathrm{~K}$, respectively, for a carrier density $\mathrm{N}$ which ranges between $10^{15}$ and $5 \times 10^{18} \mathrm{~cm}^{-3}$. In particular, curves at several representative carrier density values have been plotted: A: $1 \times 10^{15} \mathrm{~cm}^{-3}$, B: $1 \times 10^{17} \mathrm{~cm}^{-3}$, C: $6 \times 10^{17} \mathrm{~cm}^{-3}$, D: $9 \times 10^{17} \mathrm{~cm}^{-3}$, E: $2 \times 10^{18} \mathrm{~cm}^{-3}$, F: 3 $\times 10^{18} \mathrm{~cm}^{-3}$, G: $4 \times 10^{18} \mathrm{~cm}^{-3}$, H: $5 \times 10^{18} \mathrm{~cm}^{-3}$. From now on, the capital letter labels will be used to indicate the carrier density at which the curves have been calculated. The excitonic absorption peak decreases progressively for increasing carrier concentration to finally disappear at a concentration of about $7 \times 10^{17} \mathrm{~cm}^{-3}$ density at which a complete screening of the exciton is accomplished. At densities higher than 2 $\times 10^{18} \mathrm{~cm}^{-3}$, negative values of the absorption corresponding to stimulated emission over the Mott density are obtained. The calculated absorption coefficient in the absence of excitation (curve A, for a carrier density of $10^{15} \mathrm{~cm}^{-3}$ ) coincides with the experimental points reported by Piccioli ${ }^{22}$ [see the inset in the upper left of Fig. 3(a)]. On comparing the two curves, a correction factor can be deduced, which allows to calculate the absolute absorption value at any density, given that the interband matrix element is unknown.

This model has been previously used in order to adequately explain room-temperature optical nonlinearities in gallium arsenide. ${ }^{21,23}$ From the comparison of the calculated absorption coefficients at $300 \mathrm{~K}$ in GaAs and GaSe we can conclude that our results in GaSe are similar to those obtained for GaAs, with the exception that the absorption coefficient values in GaSe are almost one order of magnitude lower. On the other hand, a similar reduction of the exciton absorption peak is obtained at photogenerated carrier concentrations which are approximately one order of magnitude higher in GaSe than in GaAs.

Any absorption increment $\Delta \alpha$, relative to the value in absence of excitation, is related to the refractive index increment $\Delta n$ through the Kramers-Krönig relation, which can be easily computed from the following discrete expression:

$$
\begin{aligned}
\Delta n(\sigma)= & \frac{1}{4 \pi^{2} \sigma} \sum_{k=1}^{\mathcal{N}}\left[\left(\sigma_{k}-\sigma\right) \ln \left|\sigma_{k}-\sigma\right|-\left(\sigma_{k}+\sigma\right)\right. \\
& \left.\times \ln \left(\sigma_{k}+\sigma\right)\right] P_{k}
\end{aligned}
$$
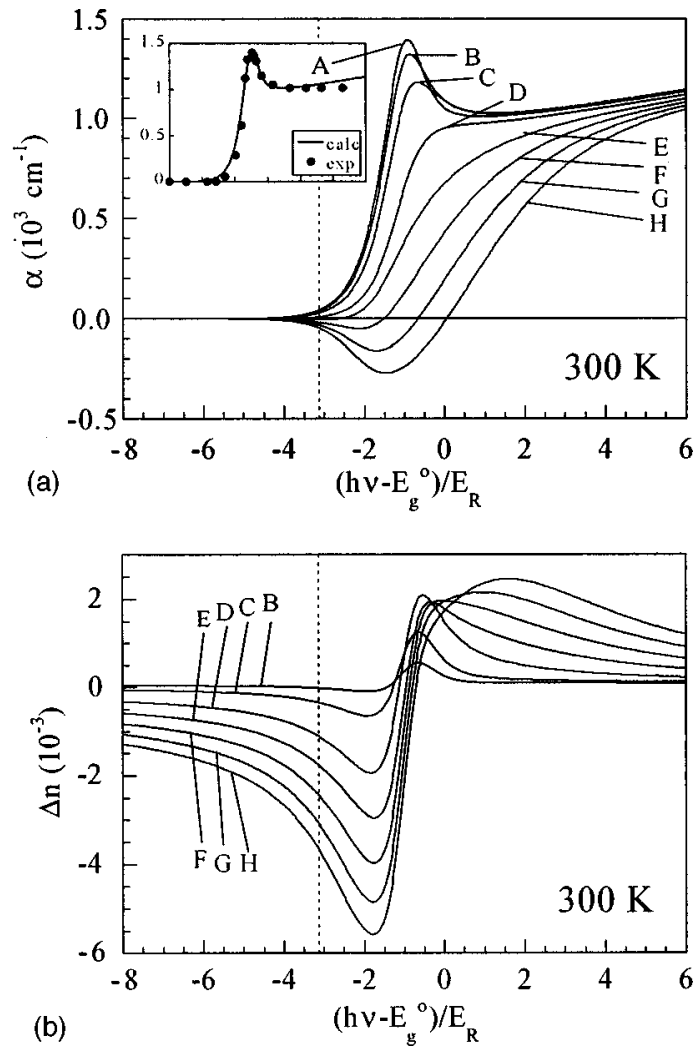

FIG. 3. (a) Calculated absorption coefficient at $300 \mathrm{~K}$ at different carrier concentrations. As stated at the beginning of Sec. IV, A: $10^{15} \mathrm{~cm}^{-3}, \mathrm{~B}$ : $10^{17} \mathrm{~cm}^{-3}$, C: $6 \times 10^{17} \mathrm{~cm}^{-3}$, D: $9 \times 10^{17} \mathrm{~cm}^{-3}$, E: $2 \times 10^{18} \mathrm{~cm}^{-3}$, F: 3 $\times 10^{18} \mathrm{~cm}^{-3}, \mathrm{G}: 4 \times 10^{18} \mathrm{~cm}^{-3}, \mathrm{H}: 5 \times 10^{18} \mathrm{~cm}^{-3}$. Inset: calculated absorption curve A $\left(N=10^{15} \mathrm{~cm}^{-3}\right)$. together with some experimental points from the literature (see Ref. 21). (b) The corresponding refractive index increments, calculated via the Kramers-Krönig relations, with absorption curve $A$ as a reference. In both figures the dashed vertical line indicates the probe wavelength position on the abcisa.

with

$$
P_{k}=\frac{\Delta \alpha\left(\sigma_{k+1}\right)-2 \Delta \alpha\left(\sigma_{k}\right)+\Delta \alpha\left(\sigma_{k-1}\right)}{\Delta \sigma}
$$

by taking in account the conditions $\Delta \alpha\left(\sigma_{1}\right)=\Delta \alpha\left(\sigma_{2}\right)$ and $\Delta \alpha\left(\sigma_{\mathcal{N}-1}\right)=\Delta \alpha\left(\sigma_{\mathcal{N}}\right)$.

Here $\sigma$ is the specific wave number at which the refractive index is computed, by calculating the sum over all the $\sigma_{k}$, whereas $\Delta \alpha(\sigma)=\alpha(\sigma, N)-\alpha\left(\sigma, N_{\text {ref }}\right)$ represents, in our case, the absorption coefficient increment when the absorption curve A in Fig. 3(a) is taken as a reference $\left(N_{\text {ref }}\right.$ $\left.=1 \times 10^{15} \mathrm{~cm}^{-3}\right)$.

Figures 3(b) and 4(b) show the refractive index increments for the corresponding $\alpha$ values in Figs. 3(a) and 4(a). There is not a $\Delta n$ curve $\mathrm{A}$ in this set of plots as it would just be a zero line.

It must be pointed out that at the probe wavelength the order of magnitude of the computed $\Delta n$ and $\Delta \alpha$ values is compatible with those deduced in the previous section from the experimental transmittance.

In order to reproduce the transmitted intensity time response, the carrier density $N$ is needed as a function of time. It has been obtained as a numerical solution to the recombination differential equation 

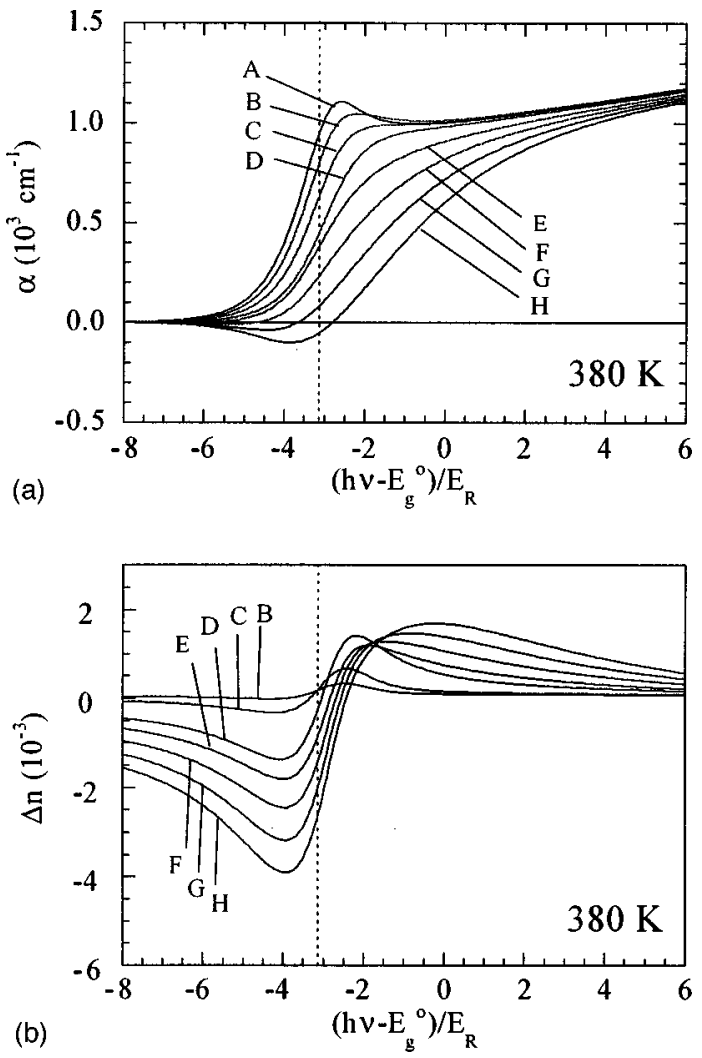

FIG. 4. (a) Calculated absorption coefficient at $380 \mathrm{~K}$ at different carrier concentrations. The dashed vertical line indicates the probe wavelength position on the abcisa. (b) The corresponding refractive index increments, calculated via the Kramers-Krönig relations, with absorption curve A as a reference. In both figures the dashed vertical line indicates the probe wavelength position on the abcisa.

$$
\frac{\partial N}{\partial t}=\alpha \phi(t)-k_{Q} N^{2}-k_{L} N,
$$

where $\alpha$ is the absorption coefficient at the pump laser wavelength $\left(\alpha=2000 \mathrm{~cm}^{-1}\right), k_{Q}$ is the quadratic recombination coefficient, $k_{L}$ is a linear recombination coefficient, and $\phi(t)$ is the pump pulse, which is best reproduced by the function

$$
\phi(t)=\phi_{0}\left(\frac{t}{\tau}\right)^{3} e^{-(t / \tau)^{3}} \quad t \geqslant 0
$$

where $\tau$ is the pulse width and $\phi_{0}=\phi_{\max } e$. In the steady state, when $\partial N / \partial t=0$, the carrier concentration, which takes its maximum value, has a square-root dependence on the absorbed photon flux, consistently with the linear behavior pointed out in the previous section and illustrated by the inset in Fig. 1. An approximate value for $k_{Q}$ is provided in the steady state by the relation $k_{Q}=\alpha \phi_{N \text { max }} /\left(N_{\text {max }}\right)^{2}$, where $\phi_{N \text { max }}$ is the absorbed photon flux at the carrier concentration maximum $N_{\max }$.

Even if the recombination is predominantly quadratic, the linear term with the coefficient $k_{L}$ has been introduced in order to guarantee that the carrier concentration vanishes at longer times $(t \gg \tau)$, when the pump pulse intensity has also substantially decreased.

Once the concentration $N(t)$ is known, $\alpha(t)$ and $n(t)$ can be calculated to give the calculated transmission pulse $T(t)$, by using Eq. (1). For practical reasons, a limited number of carrier concentration values has been considered in order to calculate the $\alpha$ and $\Delta n$ curves. The absorption coefficient and refractive index values at the probe wavelength have been plotted versus the carrier concentration and fit with a polynomial function that has been subsequently used to compute $\alpha(t)$ and $n(t)$.

Let us discuss the transmission example at $300 \mathrm{~K}$ and photon flux of $6 \times 10^{24} \mathrm{~cm}^{-2} \mathrm{~s}^{-1}$ (curve $\mathrm{g}^{\prime}$ in Fig. 1). In the previous section we deduced a maximum expected refractive index decrement $\Delta n=-0.001$, which implies a maximum carrier concentration $N_{\max } \approx 1 \times 10^{18} \mathrm{~cm}^{-3}$. Besides, $\phi_{N \text { max }}$ $\approx 0.8 \phi_{\text {max }}$, as it can be deduced from the delay between the laser pulse in Fig. 1 and the point in which the transmission pulse takes its minimum value. Such values lead to a recombination coefficient $k_{Q}$ which is too high $\left(1 \times 10^{-8}\right.$ $\mathrm{cm}^{-3} / \mathrm{s}$ ), and to an absurd carrier concentration pulse, with quickly disappearing free carriers and concentration values which subsequently become negative at an early stage. Reducing $k_{Q}$ would lead to an increment of the carrier concentration and to a decrement of the refractive index larger than that deduced from the experimental transmission pulse.

The explanation for such a result lies in the effective photogenerated carrier concentration involved in this nonlinear phenomenon. On the one hand, the pump laser intensity profile has been implicitly considered flat whereas, far from being Gaussian, it is quite inhomogeneous and presents some ' 'hot', points, as experimentally observed. Just the assumption of a Gaussian profile leads to an energy reduction ranging from $15 \%$ to $78 \%$, depending on the considered spot radius. On the other hand, a relevant diffusion might be present, with carriers running away from the area limited by the probe beam spot. Both effects can be accounted for in the code by introducing an effective energy invested in carrier generation instead of the nominal energy.

In the example taken under discussion with $3 \mathrm{~mJ}$ of nominal energy and just $1.06 \mathrm{~mJ}$ absorbed by the sample $\left(6 \times 10^{24} \mathrm{ph} \mathrm{cm}^{-2} \mathrm{~s}^{-1}\right)$ an effective energy of $0.07 \mathrm{~mJ}(\approx 4$ $\left.\times 10^{23} \mathrm{ph} \mathrm{cm}^{-2} \mathrm{~s}^{-1}\right)$ is consistent with the expected refractive index decrement and reproduces correctly the experimental transmission pulse, as shown by curve $\mathrm{g}$ in Fig. 5(a). A reduction of the absorbed energy (with a reduction factor which is kept constant) leads to calculated pulses which fit well the experimental data at lower nominal energies (curves c and f) by keeping $k_{Q}$ and $k_{L}$ constant. At $380 \mathrm{~K}$ the calculated and experimental transmission pulses are also in agreement, as shown in Fig. 5(b). It must be pointed out that in this case the same effective energies have been considered and the pulses which best fit the experimental points have been calculated using recombination coefficients which are the same order of magnitude as those introduced at $300 \mathrm{~K}$.

\section{SUMMARY}

In this article we have reported on a transmission nonlinearity which consists of a change in the $\mathrm{He}-\mathrm{Ne}$ probe beam intensity transmitted by GaSe samples in response to a $7.8 \mathrm{~ns} \mathrm{Nd}-\mathrm{YAG}$ laser beam (pump) incident pulse. At $300 \mathrm{~K}$, and after the correction for thermal effects, a $40 \%$ maximum transmittance decrement is found, consistently with a refractive index decrement $\Delta n=-0.001$. 

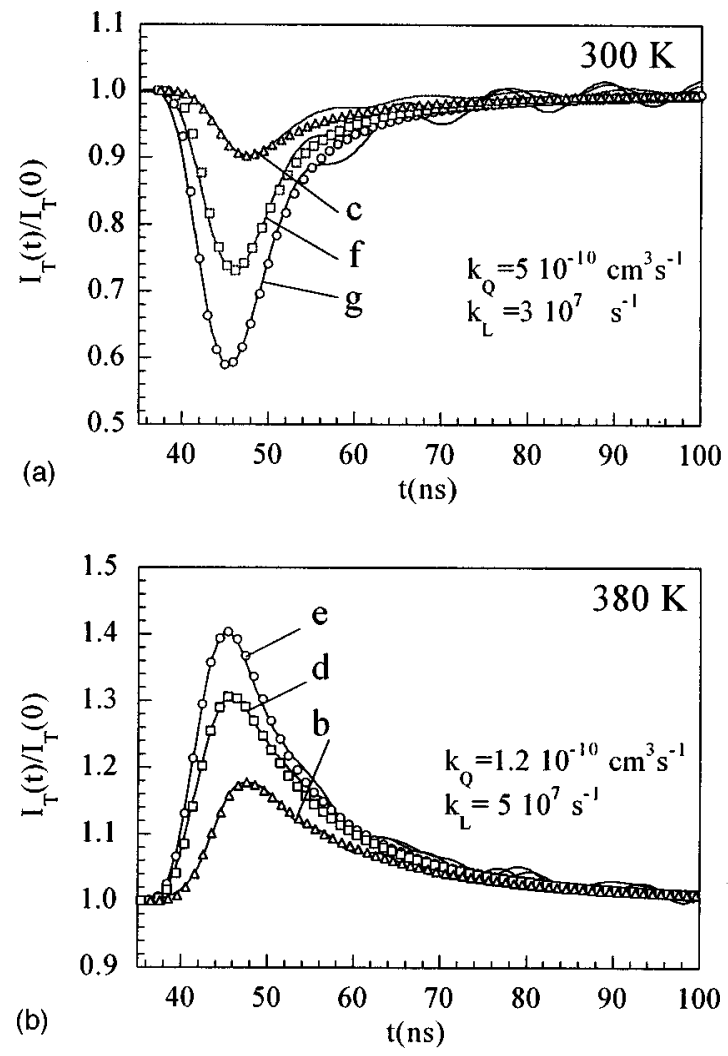

FIG. 5. (a) Experimental transmission pulses at $300 \mathrm{~K}$ corrected for thermal effects (solid lines) together with the calculated pulses (discrete points). (b) The same at $380 \mathrm{~K}$. The correspondence between labels and photon fluxes established at the beginning of Sec. III has been used, with (b) $1.4 \times 10^{24}$, (c) $2.3 \times 10^{24}, \quad$ (d) $3.6 \times 10^{24}, \quad$ (e) $5.4 \times 10^{24}, \quad$ (f) $8.5 \times 10^{24}, \quad$ (g) 17 $\times 10^{24}\left(\mathrm{ph} \mathrm{cm}^{-2} \mathrm{~s}^{-1}\right)$.

We have attributed such transmission transients to an excitonic screening by the pump photogenerated carriers, which leads to absorption bleaching and hence to a refractive index reduction. We have calculated such absorption decrement with the Bányai and Koch semiphenomenological model which incorporates a screened Coulomb potential, a gap renormalization, and a band filling term, with the carrier density as a parameter. The subsequent refractive index reduction has been computed via the Kramers-Krönig relations. The obtained $\Delta n$ and $\Delta \alpha$ values are compatible with those deduced from the experimental transmittance.

We have calculated the time response of the devices by assuming that the main recombination process is quadratic (as the linear dependence of the transmission maxima with the square root of the absorbed energy suggests) and taking into account that carrier diffusion might be present. The transmission pulses obtained with this model are in good agreement with the experimental ones at different temperatures and incident photon fluxes.

We consider that the main contribution of this article is, therefore, having identified the mechanism underlying the transmission nonlinearity, i.e., a decrement in the absorption coefficient and refractive index due to the excitonic screening by the photogenerated carriers, and having quantitatively evaluated the magnitude of such a decrement. The time re- sponse of the devices described in this article makes them suitable for modulation or commutation at frequencies over $100 \mathrm{MHz}$.

\section{ACKNOWLEDGMENT}

Financial support from the Spanish Government CICYT (Project No. MAT95-0391) is acknowledged.

${ }^{1}$ G. A. Akhundov, A. A. Agaeva, V. M. Salmanov, Yu. P. Sharonov, and Y. D. Yaroshetskii, Fiz. Tekh. Poluprovodn. FTPPA47, 1229 (1972) [Sov. Phys. Semicond. 7, 826 (1973)].

${ }^{2}$ V. I. Sokolov, Yu. F. Solomonov, and V. K. Sovashiev, Fiz. Tverd. Tela (Leningrad) FTVTAC17, 1914 (1975) [Sov. Phys. Solid State 17, 1256 (1976)].

${ }^{3}$ I. M. Catalano, A. Cingolani, A. Minafra, and C. Paorici, Opt. Commun. 24, 105 (1978).

${ }^{4}$ Yu. F. Solomonov and V. K. Sobashiev, Phys. Status Solidi A 74, 75 (1982).

${ }^{5}$ G. B. Abdullaev, K. R. Alakverdiev, M. E. Karaseev, V. I. Konov, L. A. Kulewskii, N. B. Mustafaev, P. P. Pashinin, A. M. Prokhorov, Yu. M. Starodunov, and N. I. Chapliev, Kvant. Elektron. (Moscow) KVEKA316, 725 (1989) [Sov. J. Quantum Electron. 19, 494 (1989)].

${ }^{6}$ E. Bringuier, A. Bourdon, N. Piccioli, and A. Chevy, Phys. Rev. B 49, 16971 (1994).

${ }^{7}$ K. L. Vodopyanov, L. A. Kulewskii, V. G. Voevodin, A. Y. Gribenyukov, K. R. Allakhverdiev, and T. A. Terimov, Opt. Commun. 83, 322 (1991).

${ }^{8}$ A. Bianchi, A. Ferrario, and M. Musci, Opt. Commun. 25, 256 (1978).

${ }^{9}$ J. L. Oudar, Ph. J. Kupecek, and D. S. Chemla, Opt. Commun. 29, 119 (1979).

${ }^{10}$ Ph. J. Kupecek, H. Le Person, and M. Comte, Infrared Phys. 19, 263 (1979).

${ }^{11}$ M. Wegener, C. Klingshirn, S. W. Koch, and L. Banyai, Semicond. Sci. Technol. 1, 366 (1986).

${ }^{12}$ G. P. Golubev, V. S. Dneprovskii, Z. D. Kovalyuk, and V. A. Stadnyk, Sov. Phys. Solid State 27, 265 (1985).

${ }^{13}$ A. M. Bakiev, Yu. V. Bandishev, G. S. Volkov, V. S. Dneprovskii, Z. D. Kovalyuk, A. R. Lesiv, S. V. Savinov, and A. Y. Furtichev, Sov. Phys. Solid State 28, 579 (1986).

${ }^{14}$ C. Hirlimann, J. F. Morange, M. A. Kanehisa, A. Chevy, and C. H. Brito Cruz, Appl. Phys. Lett. 55, 2307 (1989).

${ }^{15}$ V. S. Dneprovskii, A. I. Furtichev, V. I. Klimov, E. V. Nazvanova, D. K. Okorokov, and U. V. Vandishev, Phys. Status Solidi B 146, 341 (1988).

${ }^{16}$ A. Segura, J. Bouvier, M. V. Andrés, F. J. Manjón, and V. Muñoz, Phys. Rev. B 56, 1 (1997).

${ }^{17}$ R. Le Toullec, M. Piccioli, M. Mejatti, and M. Balkansky, Nuovo Cimento B 38, 159 (1977).

${ }^{18}$ J. Camassel, P. Merle, H. Mathieu, and A. Chevy, Phys. Rev. B 17, 4718 (1978).

${ }^{19}$ Ch. Ferrer, A Segura, M. V. Andrés, V. Muñoz, and J. Pellicer., J. Appl. Phys. 79, 3200 (1996).

${ }^{20}$ Landolt-Börnstein Tables, edited by O. Madelung, M. Schulz, and H. Weiss (Springer, Berlin, 1983), vol. III/17f.

${ }^{21}$ L. Bányai and S. W. Koch, Z. Phys. B 63, 283 (1986).

${ }^{22}$ N. Piccioli, Thèse de Doctorat d'Etat, Université Pierre et Marie Curie, 1987.

${ }^{23}$ Y. H. Lee, A. Chavez-Pirson, S. W. Koch, H. M. Gibbs, S. H. Park, J. Morhange, A. Jeffery, N. Peyghambarian, L. Banyai, A. C. Gossard, and W. Wiegmann, Phys. Rev. Lett. 57, 2446 (1986). 